\title{
A Relação entre o Mercado de Accões e as Variáveis Macroeconômicas: Uma Análise Econométrica para o Brasil $^{*}$
}

\author{
Maurício S. Nunes ${ }^{* *}$ \\ Newton C. A. da Costa Jr. ${ }^{* * *}$ \\ Roberto Meurer $^{* * * *}$
}

Sumário: 1. Introdução; 2. Revisão de literatura e modelos estimados; 3. Variáveis e ordem de integração; 4. Evidência empírica; 5. Conclusão.

Palavras-chave: variáveis macroeconômicas; mercado de ações; causalidade reversa; proxy effect.

Códigos JEL: E44; G10; C50.

Este artigo analisa a relação entre variáveis macroeconômicas e retornos do Ibovespa no período pós-Plano Real. Verificou-se que os retornos do mercado de ações não servem de hedge para a inflação esperada e não se constatou relação negativa entre inflação e atividade econômica. Estes resultados são inconsistentes com as hipóteses fisheriana modificada e proxy effect. Contudo, a "causalidade reversa" , que reflete a influência dos movimentos no mercado de ações sobre a taxa de inflação, medida pelas variações da taxa de juros ex-ante, foi verificada. Mostrou-se, também, que variações do Ibovespa e PIB real não apresentaram relação significativa, mas a relação entre setor externo e Ibovespa foi verificada através da taxa de câmbio real e spreads do C-Bond.

We study the relationship between a set of macroeconomics variables and stock market returns for the Brazilian economy after the Real Plan. We find that returns do not hedge against expected inflation, and the inflation rate and output show no negative relationship, which is inconsistent with a "modified fisherian" and "proxy effect" hypothesis. Yet there is "inverse causality", i.e. stock market movements affect inflation rate if measured by ex ante interest

\footnotetext{
*Artigo recebido em ago. 2004 e aprovado em abr. 2005. Os autores agradecem aos dois pareceristas anônimos pelos comentários e sugestões, responsabilizando-se, no entanto, pelos eventuais erros e omissões remanescentes.

${ }^{* *}$ Doutorando em economia no Programa de Pós-Graduação em Economia da UFRGS.

*** Professor do Programa de Pós-Graduação em Economia da UFSC e pesquisador do CNPq. ***** Professor do Programa de Pós-Graduação em Economia da UFSC.
} 
rates. Results show no significant relationship between stock market and output. We also find a relationship between the Bovespa index and C-bond spreads and exchange rates.

\section{Introdução}

Nos últimos vinte anos, o desempenho macroeconômico na maioria dos países desenvolvidos e emergentes tem melhorado substancialmente. Em ambos, a inflação e a taxa de crescimento real apresentam-se mais estáveis do que no início dos anos 80. Parte dessa evolução está relacionada ao desenvolvimento dos mercados financeiros, que contribuíram para melhorar a alocação dos recursos financeiros ao setor produtivo da economia (Bekaert et alii, 1995).

No Brasil, recentemente, o mercado de ações tem recebido muita atenção por parte de investidores e empresas, haja vista que o mesmo tem se apresentado como uma oportunidade para investidores externos que visem diversificar seus portfólios. Após a implementação do Plano Real, o mercado financeiro brasileiro apresentou um súbito desenvolvimento, de forma que a capitalização através do mercado de ações apresentou crescimento tanto em termos de volume de negócios como na eficiência alocativa.

No entanto, devido à insegurança quanto às condições macroeconômicas e a sua estrutura financeira, a capitalização por intermédio do mercado de ações no Brasil tem apresentado um grau de risco elevado. Como conseqüência, as ações negociadas na bolsa de valores brasileira ficaram vulneráveis às condições econômicas adversas, sejam elas internas ou externas. Isso acarretou diferentes percepções do risco por parte dos investidores e propiciou uma certa exposição da moeda nacional a ataques especulativos e exigiu freqüentes intervenções no mercado por parte do governo, desviando-o de sua principal atribuição que é prover as condições necessárias para viabilidade do processo de desenvolvimento econômico do país.

Neste sentido, o presente trabalho tem como objetivo verificar se há relação entre um conjunto de variáveis macroeconômicas e o retorno dos ativos no mercado de ações brasileiro. Além disso, pretende-se adicionar um fator de risco como parâmetro das expectativas dos investidores externos com relação ao mercado brasileiro.

Os resultados obtidos apontam para rejeição das hipóteses "fisheriana modificada" e "proxy effect", ou seja, os retornos do mercado de ações brasileiro não servem como um perfeito hedge para taxa de inflação esperada, assim como a relação negativa entre as taxas de inflação e o nível de atividade econômica não pôde ser constatada. Todavia, não se pode rejeitar a hipótese de "causalidade reversa". Os 
resultados indicam a influência dos movimentos no mercado de ações sobre a taxa de inflação, medida pelas variações da taxa de juros ex-ante. A relação dinâmica entre as variáveis é analisada utilizando-se uma estimativa VAR. Os resultados ratificam a significativa influência dos retornos de mercado em antecipar as variações nas taxas de inflação, assim como na taxa de câmbio. Conforme esperado, constata-se uma reação negativa das taxas de juros às oscilações no mercado de ações. Em contrapartida, as variações do índice de mercado e as variações no PIB não apresentam uma relação significativa. Por fim, verifica-se uma significativa relação negativa entre o Ibovespa e os spreads dos C-bonds, indicando que a percepção dos investidores externos em relação à economia brasileira está fortemente relacionada às oscilações no mercado de ações.

Para tanto, além desta introdução, descreve-se os modelos estimados e faz-se a revisão de literatura sobre a relação entre variáveis macroeconômicas e o mercado de ações, apresentados na próxima seção. As séries e a ordem de integração são apresentadas na terceira seção e finalmente, analisam-se os resultados e tecem-se algumas considerações finais.

\section{Revisão da Literatura e Modelos Estimados}

\subsection{A hipótese de "proxy effect"}

Uma ampla literatura financeira tem argumentado que os preços dos ativos desenvolvem-se inversamente às taxas de inflação. Essa relação negativa entre as variáveis foi destacada inicialmente a partir da hipótese de "proxy effect" desenvolvida por Fama (1981). Fama argumentava que a relação negativa entre os retornos das ações e inflação era uma proxy da relação negativa entre inflação e o nível de atividades, contrariando a relação positiva exposta na curva de Phillips. De acordo com Merikas (2002), a evidência da hipótese proxy decorre do fato que uma forte atividade econômica causa inflação e induz os policymakers a implementar políticas macroeconômicas contra-cíclicas. A resposta negativa dos preços das ações ao melhor desenvolvimento presente da economia é justificada se os efeitos esperados de uma política contracionista são superiores ao ganho esperado decorrente do aumento da produção. Isso acontece porque a melhoria da produção para o período vigente já foi prevista e, por conseguinte, incorporada aos preços dos ativos em períodos anteriores, de forma que o impacto refletido pelos preços das ações no período atual é decorrente da reação contracionista dos policymakers à elevação da taxa de inflação.

Adicionalmente, pode-se analisar a relação negativa entre as variáveis a partir 
de uma versão modificada do modelo de Fisher (1930). Extrapolando um pouco a idéia original, pode-se testar que, sob a hipótese de mercados eficientes, ${ }^{1}$ o retorno real esperado e a taxa de inflação esperada variam independentemente, de forma que, em média, os investidores sejam compensados pelas variações no poder de compra (Gutelkin, 1983).

A equação dos retornos pode ser descrita como:

$$
R_{t}=\alpha_{t}+\beta E\left(\pi_{t} / \phi_{t-1}\right)+\epsilon_{t}
$$

onde:

$R_{t}$ é o retorno nominal de mercado no período $t$;

$\pi_{t} / \phi_{t-1}$ é a taxa de inflação no período $t$ condicionada ao conjunto de informações utilizadas pelo investidor para formar suas expectativas;

$E$ é o operador de expectativa matemática; e

$\epsilon_{t}$ é um distúrbio aleatório.

O modelo disposto na equação (1) estima o valor condicional esperado dos retornos $R_{t}$ do mercado de ações como uma função da taxa de inflação esperada. Assim, uma estimativa de $\beta=1$ (ou muito próximo) é consistente com a hipótese "fisheriana modificada" de que os retornos nominais de mercado variam de forma idêntica com as variações na taxa de inflação esperada, o que implica a afirmação de que os retornos de mercado servem como um perfeito hedge contra inflação esperada. Entretanto, considerando que o retorno real esperado de um ativo é igual ao seu retorno nominal deduzida a taxa de inflação esperada, uma estimativa de $\beta=1$ é consistente com a suposição de que o retorno real esperado de um ativo e a taxa de inflação esperada variam independentemente.

\section{Estimação das hipóteses de "proxy effect" e "fisheriana modifi- cada"}

Para testar as hipóteses de "proxy effect" e "fisheriana modificada", estima-se as inflações esperada e não esperada a partir de um modelo ARIMA (autoregressivo integrado média móvel). No entanto, desde que a explicação do "proxy effect" esteja baseada na relação indireta entre o retorno das ações e a inflação, devese estimar o modelo por um procedimento de dois estágios, no qual os níveis de

\footnotetext{
${ }^{1}$ De acordo com essa hipótese, os preços dos ativos negociados em determinado mercado devem refletir todo o conjunto de informações disponíveis aos seus agentes. Neste estudo, assume-se que os mercados de capitais são quase eficientes e, portanto, trabalha-se implicitamente com a hipótese de eficiência de mercado.
} 
inflação são regredidos contra a taxa de crescimento da atividade real em níveis defasados, contemporâneo e previstos, conforme descrito:

$$
I_{t} ; E(I)_{t} ; U(I)_{t}=\sum_{i=-n}^{n} b_{i} \Delta R_{t+1}+\epsilon_{t}
$$

No segundo passo, o retorno real é regredido contra os valores defasados, contemporâneos e previstos das taxas de crescimento do nível de atividades, e os resíduos da equação 2 , como segue:

$$
R_{t}-I_{t}=\alpha_{0}+\alpha_{i} \epsilon_{t}+\sum_{i=-n}^{n} b_{i} \Delta R_{t+1}+\xi_{t}
$$

onde:

$\left(R_{t}-I_{t}\right)$ representa o retorno de mercado em termos reais;

$E(I)$ e $U(I)$ são os componentes esperados e não esperados da inflação, respectivamente;

$\epsilon_{t}$ representa a inflação já eliminados os efeitos da taxa de crescimento da atividade real; e

$\xi_{t}$ é um ruído branco.

\subsection{A hipótese de "causalidade reversa"}

A contrapartida à hipótese de Fama foi desenvolvida por Geske e Roll (1983). Os autores ampliaram a hipótese de "proxy effect" envolvendo o setor governamental e suas políticas monetária e fiscal, além de levantar a hipótese de "causalidade reversa", na qual o mercado de ações sinaliza as variações no processo inflacionário. O pressuposto básico é a inversão quanto às expectativas sobre a inflação esperada. Geske e Roll argumentam que os agentes adaptam suas expectativas quanto à inflação, em virtude das modificações econômicas antecipadas pelas variações nos preços dos ativos, e que essa reversão da causalidade seria caracterizada por um conjunto de eventos macroeconômicos, com destaque à interação do processo de geração de receita do governo e as variações dos preços dos ativos. Assim, quando os preços das ações declinam em resposta às variações antecipadas de condições econômicas adversas, há uma forte tendência da ocorrência de déficit governamental. Considerando, por exemplo, que o governo monetize suas dívidas e que os indivíduos antecipem essa monetização, a taxa de inflação deve aumentar. Dessa forma, as variações nos preços dos ativos, causados por variações antecipadas nas 
condições econômicas, serão correlacionadas com as variações na taxa de inflação esperada e, conseqüentemente, na taxa de inflação.

\section{Estimação da hipótese de "causalidade reversa"}

Para estimar a hipótese de "Causalidade Reversa" formulada por Geske e Roll (1983), utiliza-se um modelo de função de transferência do tipo ARIMA desenvolvido por Solnik (1983), o qual determina um estimador ótimo da taxa real esperada condicionada aos valores passados das taxas de juros, inflação e retornos de mercado. O modelo a ser ajustado é:

$$
\rho_{t}-\rho_{t-1}=\omega_{0} R_{t}+\eta_{t}
$$

onde:

$\left(\rho_{t}-\rho_{t-1}\right)$ é a variação na taxa de juros real;

$R_{t}$ é o retorno de mercado em termos reais; e

$\eta_{t}$ é um modelo de ruído com choques aleatórios $\mu_{t}$ que seguem um processo média móvel de segunda ordem sem tendência, $M A(2)$ :

$$
\eta_{t}=\theta_{1} \mu_{t-1}+\theta_{2} \mu_{t-2}+\epsilon_{t}
$$

Ajustando a equação (5), gera-se o modelo de expectativas das taxas de juros reais ex ante, da forma:

$$
\rho_{t}^{e}=\omega_{0} R_{t-1}+\left(1-\theta_{1}\right) \mu_{t-1}+\theta_{2} \mu_{t-2}
$$

onde:

$\rho_{t}^{e}$ é a taxa de juro real esperada;

$R_{t}$ o retorno real de mercado;

$\mu_{t}$ 's os componentes autoregressivos do ruído da equação de transferência; e $\omega_{0}$ e $\theta$ 's são os coeficientes.

\subsection{Estudos empíricos sobre as hipóteses de "proxy effect", "cau- salidade reversa" e "fisheriana modificada"}

Em termos empíricos, uma ampla gama de trabalhos foi desenvolvida acerca das hipóteses de "proxy effect" e "causalidade reversa", com destaque aos descritos na tabela 1. 
Tabela 1

"Proxy effect" e "causalidade reversa" - evidências empíricas

\begin{tabular}{|c|c|c|c|c|}
\hline Autor & Hipótese & Período & Local & Resultados \\
\hline $\begin{array}{l}\text { Mandelker e Tan- } \\
\text { don (1985) }\end{array}$ & "Proxy effect" & $\begin{array}{l}\text { 1966-1979 } \\
\text { (observações } \\
\text { trimestrais) }\end{array}$ & $\begin{array}{l}\text { EUA, Ca- } \\
\text { nadá, Japão, } \\
\text { Reino Unido, } \\
\text { França e Bel- } \\
\text { gica }\end{array}$ & $\begin{array}{l}\text { Aceitação. Relação ne- } \\
\text { gativa entre preços dos } \\
\text { ativos e as taxas de in- } \\
\text { flação esperada e não- } \\
\text { esperada. }\end{array}$ \\
\hline $\begin{array}{l}\text { Najand e Noronha } \\
\text { (1998) }\end{array}$ & "Proxy effect" & $\begin{array}{l}\begin{array}{l}1977-1994 \\
\text { servações } \\
\text { sais })\end{array} \\
\end{array}$ & Japão & $\begin{array}{l}\text { Aceitação. Relação cau- } \\
\text { sal direta da inflação aos } \\
\text { retornos de mercado. }\end{array}$ \\
\hline $\begin{array}{l}\text { Gallagher e Taylor } \\
(2002)\end{array}$ & "Proxy effect" & $\begin{array}{l}\text { 1957-1997 (ob- } \\
\text { servações men- } \\
\text { sais e trimes- } \\
\text { trais) }\end{array}$ & EUA & $\begin{array}{l}\text { Aceitação parcial. } \\
\text { Relação negativa entre } \\
\text { retornos dos ativos e } \\
\text { inflação depende da } \\
\text { fonte da inflação: oferta } \\
\text { ou demanda. }\end{array}$ \\
\hline Geske e Roll (1983) & $\begin{array}{l}\text { "Proxy effect" e "Cau- } \\
\text { salidade Reversa" }\end{array}$ & $\begin{array}{l}1947 / 1-1980 / 1 \\
\text { (observações } \\
\text { trimestrais) }\end{array}$ & EUA & $\begin{array}{l}\text { Rejeição da hipótese de } \\
\text { "proxy effect" e for- } \\
\text { mulação da hipótese de } \\
\text { "causalidade reversa". }\end{array}$ \\
\hline Solnik (1983) & $\begin{array}{l}\text { "Causalidade Reversa" } \\
\text { e Suposição "Fisheriana } \\
\text { Modificada" }\end{array}$ & $\begin{array}{l}\begin{array}{l}1971-1980 \\
\text { servações } \\
\text { sais })\end{array} \\
\text { men- }\end{array}$ & $\begin{array}{l}\text { EUA, Japão, } \\
\text { Inglaterra, } \\
\text { Alemanha, } \\
\text { França, } \\
\text { Holanda, } \\
\text { Bélgica, } \\
\text { Suíça e } \\
\text { Canadá }\end{array}$ & $\begin{array}{l}\text { Rejeição da hipótese } \\
\text { "fisheriana modifi- } \\
\text { cada" e aceitação da } \\
\text { hipótese de "causalidade } \\
\text { reversa". }\end{array}$ \\
\hline
\end{tabular}

\subsection{Mercado de ações, nível de atividades, taxas de juros e de câmbio: revisão empírica}

Vários estudos analisaram a relação de outras variáveis macroeconômicas relevantes e os preços dos ativos, tais como nível de atividade econômica, taxa de juros e taxa de câmbio. Em termos teóricos pode-se destacar os trabalhos de Blanchard (1990), Hansen e Singleton (1983), Campbell (1993) e Dornbush e Fischer (1980). Blanchard analisou a relação entre os preços dos ativos e a atividade econômica, com o objetivo de caracterizar a interação entre as variáveis e verificar a hipótese conjunta em relação às possíveis alterações nas políticas fiscal e monetária. O autor conclui que o mercado de ações não é a causa de um aumento na produção, ambos são resultados de variações das políticas econômicas sob certas condições vigentes na economia, pois, usualmente, um anúncio de políticas econômicas conduz à variação nas taxas de desconto e lucros antecipados que, por sua vez, levam a variações nos preços dos ativos. 
Hansen e Singleton (1983) e Campbell (1993) analisaram a relação entre preços dos ativos e taxas de juros relacionando-os com o nível de consumo intertemporal de um investidor. Os autores verificaram que o valor dos retornos futuros esperados é inversamente proporcional às expectativas sobre o fator de desconto estocástico.

Por fim, Dornbush e Fischer (1980), ao analisarem a relação entre as taxas de câmbio e a conta corrente, enfocaram a relação entre o mercado de ações e a taxa de câmbio. Considerando uma relação positiva da produção e dos níveis de riqueza e o desenvolvimento dos preços dos ativos, os autores concluíram que a elevação dos preços externos comparados aos domésticos aumenta a competitividade das empresas nacionais no mercado externo, o que refletirá na valorização de suas ações. Igualmente, considerando que os indivíduos apliquem parte de sua riqueza no mercado doméstico, uma elevação da riqueza aumentará a demanda por ações neste mercado, elevando, assim, os preços dos ativos.

Em termos empíricos, Fama (1981), Kaul (1987), Barro (1990) e Shah (1989), por exemplo, concluíram que grandes frações (superiores a $50 \%$ ) das variações nos retornos anuais poderiam ser utilizadas para prever as variáveis econômicas reais como o PIB real, a produção industrial e os níveis de investimentos, que são determinantes importantes dos fluxos de caixa das firmas. Entretanto, Kwon e Shin (1999), concluiram que, apesar de as variações nos preços dos ativos no mercado coreano serem cointegradas com um conjunto de variáveis macroeconômicas, ${ }^{2} \mathrm{o}$ índice de mercado não é um bom indicador antecedente das variáveis econômicas, o que é inconsistente com a hipótese de que o mercado de ações sinaliza a mudança de sinal apresentada pelo nível de atividades (Fama, 1990).

Em relação à taxa de juros, Rigobon e Sack (2001) analisaram a reação da política monetária americana às variações no mercado de ações entre 1985 e 2000. Os resultados encontrados sugerem que os movimentos no mercado de ações têm um significante impacto nas taxas de juros de curto prazo. Essa resposta é condizente com os efeitos dos movimentos nos preços dos ativos sobre a demanda agregada, sugerindo, portanto, que os policymakers estão reagindo às variações no mercado para evitarem possíveis impactos negativos sobre a economia. ${ }^{3}$

A relação entre taxa de câmbio e preço dos ativos é destaque nos trabalhos de Ajayi et alii (1998) e Lee e Solt (2001). Os primeiros encontraram uma relação causal unidirecional (no sentido de Granger) dos retornos dos ativos para taxa

\footnotetext{
${ }^{2} \mathrm{O}$ conjunto de variáveis macroeconômicas foi composto pelo índice de produção, taxa de câmbio, balança comercial e oferta de moeda. O período estudado foi de 1980-1992 com observações mensais.

${ }^{3}$ Um exemplo disso é a formação de uma "bolha" especulativa que, ao ser estourada, pode acarretar conseqüências adversas à economia.
} 
de câmbio para as economias desenvolvidas. ${ }^{4}$ No segundo, os autores verificaram que os excessos dos retornos em termos reais refletem as variações nos termos de troca real, bem como variações reais da taxa de câmbio induzidas por fatores macroeconômicos na Alemanha, EUA e Japão.

\subsection{Variáveis macroeconômicas e retornos no mercado de ações: estimativa $\mathrm{VAR}^{5}$}

A relação dinâmica entre as variáveis é investigada via um processo VAR, no sentido de verificar suas interações. Na estimativa, restringe-se o relacionamento entre os termos de distúrbios a partir de um processo de Innovation Accounting (Função de Resposta ao Impulso e Decomposição da Variância). ${ }^{6}$ A decomposição da variância dos erros de previsão permite inferências extraídas, considerando a proporção dos movimentos em uma série particular em virtude do seu próprio choque inicial vis-a-vis os choques oriundos das demais variáveis do sistema VAR. A análise da função de resposta ao impulso, por sua vez, representa o horizonte temporal dos efeitos dos choques das demais variáveis no VAR sob uma variável particular (Enders, 1995).

A equação final pode ser representada por:

$$
Z_{t}=\sum_{s=0}^{\infty} \beta(s) G^{-1} G \epsilon(t-s)=\sum_{s=0}^{\infty} \beta(s) G^{-1} G \mu(t-s)=\sum_{s=0}^{\infty} C \mu(t-s)
$$

onde os coeficientes $C(s)$ representam as repostas aos choques ou inovações em determinada variável particular, e pode-se alocar a variância de cada elemento em $Z_{t}$ (um vetor $m \times 1$ com $m \geq 2$ ) por fontes dos elementos de $\mu$, haja vista que $\mu$ é serialmente e contemporaneamente não correlacionado. Esta ortogonalização pode também prover:

$$
\sum_{s=0}^{t-1} C_{i j}(s)^{2} / \sum_{j=1}^{m} \sum_{s=0}^{t-1} C_{i j}(s)^{2}
$$

\footnotetext{
${ }^{4}$ Os mercados avançados foram representados pelo Canadá, Alemanha, França, Itália, Japão, Inglaterra e Estados Unidos. O período estudado compreende observações mensais de 1985 a 1991.

${ }^{5}$ No presente trabalho, a derivação do modelo VAR tomará como base para as especificações de Lee (1992).

${ }^{6}$ Expressão criada por Sims (1980) e analisa a correlação entre as inovações das respectivas variáveis.
} 
A qual representa o componente da variância do erro de previsão de $Z_{i}, t$ passos à frente, que é considerado pelas inovações em $z_{j}$.

\section{Variáveis e Ordem de Integração}

\subsection{Determinação das variáveis}

Conforme mencionado, a proposta do trabalho é verificar a relação entre um conjunto de variáveis macroeconômicas e os retornos no mercado de ações brasileiro no período de jan/1995 a dez/2004. Para tanto, optou-se pela seleção de variáveis supostas como importantes fatores macroeconômicos na determinação dos preços dos ativos negociados no mercado de ações brasileiro. Selecionaram-se como variáveis relevantes à análise o PIB, as taxas de juros, câmbio e inflação, os retornos médios de mercado e o spread do C-bond.

O PIB é a variável mais utilizada quando se objetiva avaliar o nível de atividade econômica de um país, mas apresenta traços de sazonalidade quando observado em freqüência inferior à anual. Dessa forma, optou-se, pela utilização da série do PIB mensal do Banco Central do Brasil, dessazonalizado utilizando o programa X-12-ARIMA do U.S. Census Bureau.

O nível de preços é o Índice Geral de Preços-Mercado (IGP-M). ${ }^{7}$ A taxa de câmbio real foi calculada multiplicando-se a taxa de câmbio nominal pelo nível de preços externos, representado pelo Producer Price Index (PPI) americano, e dividido pelo IGP-M.

O principal instrumento de política monetária à disposição do Banco Central são as operações de mercado aberto, que lhe permitem controlar as taxas de juros do mercado monetário. Nesse sentido, optou-se por utilizar a taxa SELIC, como uma proxy da taxa de juros.

Para representar os retornos do mercado de ações, utilizou-se o índice de mercado da Bolsa de Valores de São Paulo - Ibovespa. Para captar as percepções de risco por parte dos investidores externos, foi utilizada a série do spread entre o C-bond (título da dívida externa brasileira) e o título da dívida americana de mesma maturidade, cuja série é disponibilizada pelo Ipeadata.

Como a literatura financeira dá destaque à influência dos períodos de crises financeiras e quebras estruturais sobre os mercados financeiros (Schwert, 1989), testa-se essa influência através da utilização de variáveis dummy. Foram utilizadas dummies para representar as crises asiática (1997:11), russa (1998:09), mudança

\footnotetext{
${ }^{7}$ Utilizou-se o IGP-M como índice de preços por ser o principal referencial no mercado financeiro brasileiro.
} 
de regime cambial ocorrida em janeiro de 1999, bem como o efeito das eleições (2002:4 - 2002:11).

As séries de PIB, Ibovespa e juros foram deflacionadas pelo IGP-M. A periodicidade dos dados é mensal, com período de cobertura entre 1995:1 e 2004:12, em um total de 120 observações. $^{8}$

\subsection{Ordem de integração}

A investigação formal das propriedades de integração das séries foi conduzida aplicando-se, primeiramente, os testes de raiz unitária convencionais como DickeyFuller aumentado (ADF), e Phillips-Perron (PP), apresentados na tabela 2. ${ }^{9}$

Os resultados dos testes convergem indicando que, com exceção da taxa de juros real (estacionária em nível), todas as variáveis são não estacionárias em nível e estacionárias em primeira diferença. Portanto, pode-se afirmar que as séries referentes ao índice de mercado (Ibovespa) em termos reais, PIB real, as taxa de inflação e de câmbio real e os spreads do C-bond são todas integradas de primeira ordem - i.e. $\mathrm{I}(1)$.

Tabela 2

Teste de raiz unitária

\begin{tabular}{lcccccccc}
\hline \multicolumn{9}{c}{ Nível } \\
\hline Variáveis & ADF & Lags $^{\mathrm{a}}$ & $\mathrm{PP}^{\mathrm{b}}$ & $\tau_{\text {crit }}{ }^{c}$ & ADF & Lags $^{\mathrm{a}}$ & PP $^{\mathrm{b}}$ & $\tau_{\text {crit }}{ }^{\mathrm{a}}$ \\
\hline IBOVR & $-2,20^{*}$ & 4 & $-1,66^{*}$ & $-2,89$ & $-11,43$ & 0 & $-11,4$ & $-1,94$ \\
PIBR & $-3,13^{* *}$ & 6 & $-2,60^{* *}$ & $-3,45$ & $-8,80^{*}$ & 3 & $-10,83$ & $-2,89$ \\
TXCR & $-1,63$ & 1 & $-1,5$ & $-1,94$ & $-11,42$ & 1 & $-11,54$ & $-1,94$ \\
INF & 3,41 & 0 & 6,74 & $-1,94$ & $-4,82^{*}$ & 0 & $-4,85^{*}$ & $-2,89$ \\
TXJR & $-7,72^{* *}$ & 0 & $-7,92^{*}$ & $-3,45$ & - & - & - & - \\
SCBOND & $-2,33^{*}$ & 4 & $-2,39$ & $-2,89$ & $-7,74$ & 0 & $-7,7$ & $-1,94$ \\
\hline
\end{tabular}

IBOVR representa os retornos de mercado em termos reais; TXCR é a taxa de câmbio real; INF representa a taxa de inflação; TXJR é a taxa de juros real; PIBR representa a variação do PIB em termos reais e SCBOND representa os spreads do C-bond em relação aos títulos do tesouro americano. a Valor ótimo de defasagens selecionadas de acordo com o critério de Schwarz. b PP é o teste de Phillips-Perron. c Valores críticos com 5\% de significância. *Inclui constante. ${ }^{* *}$ Inclui constante e tendência. Obs.: Os testes ADF e PP foram realizados conforme especificados nos manuais de econometria, estimando, inicialmente, o modelo irrestrito com a inclusão do processo autoregressivo o termo constante e tendência.

\footnotetext{
${ }^{8}$ Os primeiros seis meses do Plano Real foram excluídos, pois, nesse período, as taxas de inflação apresentavam-se ainda relativamente altas, fruto da influência das expectativas inflacionárias por parte dos agentes.

${ }^{9}$ Adicionalmente, utilizou-se testes de raiz unitária com quebras estruturais, cujos resultados foram os mesmos que os obtidos pelos testes usuais.
} 


\section{Evidência Empírica}

Estima-se, inicialmente, a hipótese de "proxy effect", conforme disposto nas tabelas $3-5$. A partir do modelo descrito na seção 2 , pode se explicar a taxa esperada de inflação através de um modelo $\mathrm{AR}(1)$, conforme exposto na tabela 3 .

Tabela 3

Equação da Inflação - AR(1)

\begin{tabular}{l}
$\mathrm{INF}_{t}=0,8339 \mathrm{INF}_{t-1^{*}}+\epsilon_{t}$ \\
$\quad(16,36)$ \\
$\mathrm{R}^{2}$ ajustado $=0,3877 \quad \mathrm{DW}=2,01$ \\
\hline $\mathrm{INF}^{\mathrm{INF}} \mathrm{IN-1}_{t-1}$ são as taxas de inflação e o seu processo au- \\
toregressivo de $1^{\text {a }}$ ordem, *representa significância a $5 \%$. \\
Os valores entre parênteses representam a "estatística $t$ "; \\
DW é a estatística Durbin-Watson; $\mathrm{R}^{2}$ é o coeficiente de \\
determinação. Obs.: Os testes $\mathrm{LM}$ e White nos resíduos \\
da regressão indicaram para rejeição da hipótese nula de \\
correlação serial e heteroscedasticidade, respectivamente.
\end{tabular}

Conforme prevê o modelo, estimou-se a taxa de inflação e suas componentes esperada e não esperada (resíduo da regressão 9), contra as taxas de crescimento do PIB em termos reais (tabela 4).

Tabela 4

Inflação Esperada e Não Esperada - PIB

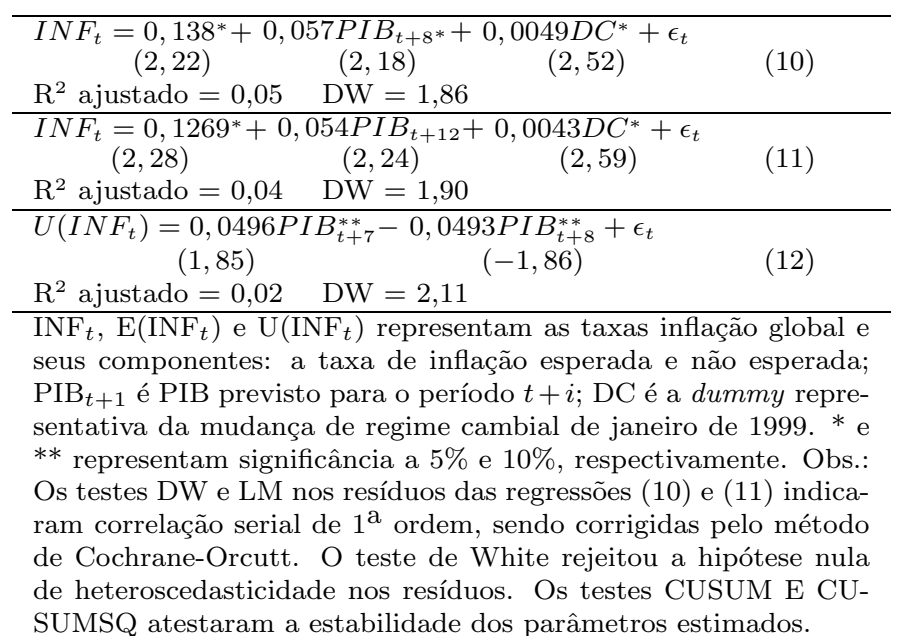

Os sinais positivos encontrados nos coeficientes da taxa de crescimento do PIB na tabela 4 tendem a rejeitar a hipótese de "proxy effect" em favor da relação 
inerente à curva de Phillips. Na equação (12), os coeficientes apresentaram sinais opostos. Nesse sentido, analisando-se tais coeficientes de forma líquida, ${ }^{10}$ rejeitase a hipótese de "proxy effect". Adicionalmente, pode se verificar que as variações futuras no nível de atividades no Brasil (durante o período estudado) podem ser antecipadas pela taxa de inflação, conforme se pôde constatar pela significância estatística dos coeficientes previstos do PIB, apesar de não haver consistência entre as regressões sobre quantos períodos o nível de inflação possa antecipar as variações sobre a atividade econômica.

No próximo passo, ressaltam-se as duas principais partes do escopo da hipótese "proxy effect", ou seja, a relação entre os retornos de mercado, taxas de inflação esperada e não-esperada e os níveis de atividade atual e futuro.

Tabela 5

Equação dos retornos do Ibovespa

\begin{tabular}{|c|c|}
\hline $\begin{array}{l}I B O V_{t}=-0,957(I N F)-0,512 P I B_{t+1 * *}+0,514 P I B_{t+7^{* *}}+\epsilon_{t} \\
(-0,63) \\
\mathrm{R}^{2} \text { ajustado }=0,10 \quad \mathrm{DW}=2,16\end{array}$ & (13) \\
\hline $\begin{array}{l}I B O V_{t}=-1,021 E(I N F)_{t}^{*}-0,0681 P I B_{t+10^{* *}+0,0699 P I B_{t+11}^{* *}+\epsilon_{t}} \\
(-2,06) \\
\mathrm{R}^{2} \text { ajustado }=0,06 \quad \mathrm{DW}=2,20\end{array}$ & (14) \\
\hline $\begin{array}{c}\begin{array}{l}U\left(I B O V_{t}\right)=-0,532 U(I N F) \\
(-1,27)\end{array} \quad \begin{array}{c}\left(-503 P I B_{t+1}^{* *}+0,0504 P I B_{t+11}^{* *}+\epsilon_{t}\right. \\
(-1,94)\end{array} \\
\mathrm{R}^{2} \text { ajustado }=0,03 \quad \mathrm{DW}=2,15\end{array}$ & (15) \\
\hline $\begin{array}{l}\left.\mathrm{INF}_{t}, \mathrm{E}\left(\mathrm{INF}_{t}\right) \text { e U(INF }{ }_{t}\right) \text { representam as taxas inflação global e seus } \\
\text { tes: a taxa de inflação esperada e não esperada, excluídas dos efeitos } \\
\text { do PIB; PIBt+i são as variações dos PIB's previstos. Obs.: Os testes L } \\
\text { nos resíduos das regressões indicaram para rejeição da hipótese nula de } \\
\text { serial e heteroscedasticidade, respectivamente. Identicamente, os test } \\
\text { e CUSUMSQ atestaram a estabilidade dos parâmetros estimados. }\end{array}$ & $\begin{array}{l}\text { onen- } \\
\text { iação } \\
\text { White } \\
\text { lação }\end{array}$ \\
\hline
\end{tabular}

A análise das equações da tabela (5) implica a negação da hipótese "fisheriana modificada" de que o mercado de ações brasileiro atuaria como um perfeito hedge contra a inflação esperada. Na equação (14), o coeficiente da taxa de inflação apresentara uma significativa relação negativa entre os retornos de mercado e a inflação esperada, sugerindo que os preços no mercado de ações brasileiro não refletem todas as informações econômicas incorporadas pela taxa de inflação esperada.

\footnotetext{
${ }^{10}$ Entende-se por análise dos coeficientes de forma líquida o somatório dos coeficientes estimados para a variável independente sob análise, nesse caso a previsão da taxa de crescimento do PIB.
} 
Igualmente, pode-se negar a existência do "proxy effect", visto que a relação positiva entre as taxas de inflação e as variações futuras no nível de atividades encontrada na tabela 4 e as relações de ambas as variáveis e o mercado de ações dispostas na tabela 5. A negação do "proxy effect" rejeita a afirmação de Fama de que a taxa de inflação esperada entra na equação dos retornos como função das taxas de crescimento futuro da atividade econômica que são as preocupações relevantes do mercado de ações. Isso significa que o mercado de ações brasileiro (no período estudado) não reflete as informações que a taxa de inflação esperada possa ter sobre a atividade econômica futura.

Analisando-se a hipótese de causalidade reversa via modelo de função de transferência (tabela 6), pode-se constatar a influência dos movimentos no mercado de ações sobre a taxa de juros em termos reais. Apesar de o coeficiente ser relativamente pequeno $(-0,11)$, esse resultado é condizente com os obtidos por Solnik para a economia japonesa $(-0,19)$, país que apresenta uma forte influência das variações no mercado de ações sobre a taxa de juros real. ${ }^{11}$ Dessa forma, considerandose a hipótese de "causalidade reversa", cujo coeficiente $\omega_{0}$ representa o impacto marginal dos retornos de mercado nas taxas de juros via um conjunto de ligações macroeconômicas, a significância desse coeficiente indica que a elasticidade da taxa de juros real às condições econômicas é sinalizada pelas variações nos preços dos ativos.

Tabela 6

Causalidade reversa (função de transferência)

\begin{tabular}{l}
$E \rho_{t}=-0,11 I B O V_{t-1 *}-0,158 \mu_{t-1 *-0,402 \mu_{t-2^{*}}+\epsilon_{t}}(-2,88) \quad(-4,71)$ \\
$\mathrm{R}^{2}$ ajustado $=0,16 \quad \mathrm{DW}=1,92 \quad(16)$ \\
\hline$E \rho_{t}$ representa a taxa de juros esperada no período $t ;$ IBOVR $_{t-1}$ \\
representa as variações nos retornos do Ibovespa no período $t-1$, \\
$\mu_{t-1}$ e $\mu_{t-2}$ representam os resíduos da função de transferência \\
nos períodos $t-1$ e $t-2$, respectivamente. Cabe ressaltar que o \\
coeficiente estimado de $\mu_{t-1}$ é $\left(1-\theta_{1}\right)$.
\end{tabular}

Quanto ao componente média móvel, observa-se que seus coeficientes apresentaram-se significativos, e com resultado líquido diferente da unidade. Esse resultado contraria os resultados de Solnik de que a taxa de juros real segue um caminho aleatório $\left(\left(1-\theta_{1}\right)+\theta_{2}=1\right)$ ao ser removido o efeito do mercado de ações sobre a mesma. Isso indica que, no Brasil, a inflação possui impacto sobre a taxa de juros real. ${ }^{12}$ Por fim, estima-se um modelo VAR, através da decomposição da

\footnotetext{
${ }^{11}$ Os demais países apresentaram coeficientes significativos, porém com valores inexpressivos (próximo de zero).

${ }^{12}$ Os resultados de Solnik foram consistentes em sete dos nove países estudados. As exceções
} 
variância dos erros de previsão e a função de resposta ao impulso entre as variáveis estudadas, conforme destacado na tabela $(7)$ e as figuras $(1-5) .{ }^{13}$

Tabela 7

Decomposição da variância dos erros de previsão das séries IBOVR, TXCR, TXJR, INF, PIBR e SCBOND

\begin{tabular}{|c|c|c|c|c|c|c|}
\hline \multirow{2}{*}{$\begin{array}{l}\text { Variáveis } \\
\text { explicadas }\end{array}$} & \multicolumn{6}{|c|}{ Decomposição na variável } \\
\hline & IBOVR & TXCR & TXJR & INF & PIBR & SCBOND \\
\hline & (\%) & (\%) & $(\%)$ & (\%) & $(\%)$ & (\%) \\
\hline IBOVR & 90,84 & 0,66 & 1,05 & 4,99 & 1,17 & 1,29 \\
\hline TXCR & 19,82 & 70,2 & 0,97 & 1,86 & 0,67 & 5,48 \\
\hline TXJR & 2,06 & 6,77 & 89,08 & 0,2 & 0,11 & 1,78 \\
\hline INF & 21,13 & 18,92 & 26,33 & 38,77 & 1,88 & 2,87 \\
\hline PIBR & 8,83 & 3,27 & 5,53 & 3,34 & 78,22 & 0,81 \\
\hline SCBOND & 54,06 & 2,39 & 3,06 & 4,21 & 0,42 & 35,86 \\
\hline \multicolumn{7}{|c|}{$\begin{array}{l}\text { IBOVR representa os retornos de mercado em termos reais; TXCR é } \\
\text { a taxa de câmbio real; INF representa a taxa de inflação; TXJR é a } \\
\text { taxa de juros real; PIBR representa a variação do PIB em termos reais } \\
\text { e SCBOND representa os spreads do C-bond em relação aos títulos } \\
\text { do tesouro americano. A variância do erro de previsão para doze } \\
\text { meses foi calculada utilizando-se um sistema VAR com } 6 \text { variáveis, } \\
\text { constantes e duas defasagens. Obs.: Foram realizados testes de di- } \\
\text { agnósticos para verificar a congruência e a estabilidade do VAR esti- } \\
\text { mado. O sistema VAR apresentou estabilidade, i.e. as raízes inversas } \\
\text { do polinômio AR ficaram dentro do círculo unitário. A congruência } \\
\text { foi verificada através dos testes LM, White e Doornik-Hansen para } \\
\text { a correlação serial, heteroscedasticidade e normalidade dos resíduos, } \\
\text { respectivamente. As defasagens no modelo foram escolhidas conforme } \\
\text { os critérios Schwarz e Hannan-Quinn, que indicaram duas defasagens. }\end{array}$} \\
\hline
\end{tabular}

Em referência ao relacionamento entre os retornos das ações e a taxa de inflação, constata-se que a segunda variável pode ser explicada pela primeira. Isto é, mesmo na presença das demais variáveis no sistema VAR, aproximadamente 21,13\% da variância dos erros de previsão para doze meses da inflação é explicada pelas inovações dos retornos do Ibovespa. Em contrapartida, menos de 5\% dos choques na inflação têm impacto nos retornos do mercado de ações brasileiro no período pós Plano Real. Esses resultados confirmam a hipótese de "causalidade reversa", mas contrariam os resultados de Schwert (1989) para a economia americana. $^{14}$

foram os EUA e Bélgica, os quais apresentaram uma certa influência das taxas de inflação no período anterior sobre as taxas de juros reais.

${ }^{13}$ As funções de resposta ao impulso foram estimadas por um processo de impulsos generalizados conforme descrito por Pesaran e Shin (1998), que dispensa a necessidade de ordenação das variáveis.

${ }^{14}$ Schwert concluiu que não há relação entre volatilidades da inflação e do mercado de ações. Os choques em ambas as variáveis tiveram um impacto insignificante (menos de $3 \%$ ) sobre aquela 
Identicamente, a relação negativa entre inflação e retorno das ações pôde ser comprovada averiguando-se a figura 1 . Um choque de um desvio padrão causa um impacto negativo de aproximadamente 0,50 ponto percentual sobre a taxa de inflação até o segundo mês após o choque, tendo seu efeito reduzido gradativamente, mas persistindo no decorrer dos doze meses. Por sua vez, um choque inesperado na taxa inflação possui uma influência moderada sobre o Ibovespa (aproximadamente, $0,30 \mathrm{pp}$ ), voltando rapidamente ao seu nível inicial e até obtendo uma pequena relação positiva no segundo mês, mas extinguindo-se completamente próximo ao terceiro mês.

Figura 1

Função de resposta - IBOVR x INF

Resposta à Inovação de Um desvio Padrão
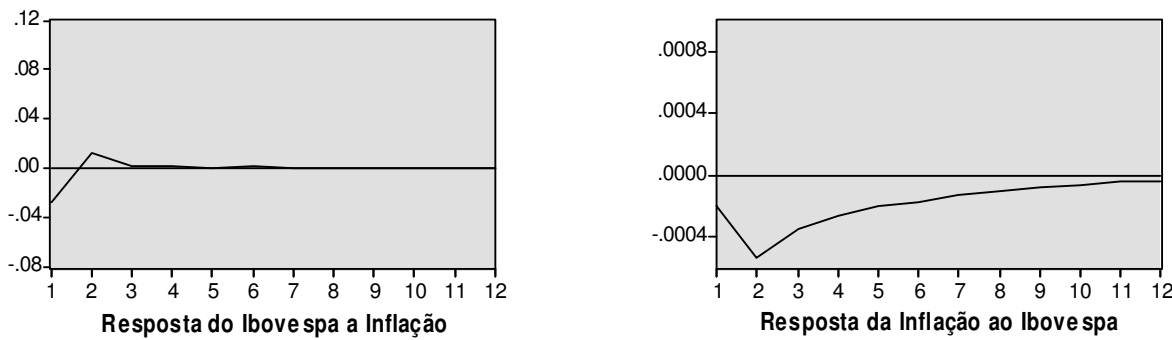

Em relação à taxa de juros, pôde-se verificar que na presença das demais variáveis macroeconômicas no modelo VAR, um choque inesperado no Ibovespa possui um impacto pouco significativo sobre a taxa de juros real (2,06\%). Identicamente, constatou-se que a taxa de juros praticamente não influencia as variações nos retornos de mercado (1,05\%). Esses resultados contrariam os indicados no modelo de função de transferência, no qual os retornos reais do Ibovespa tinham impacto significativo nas variações da taxa Selic em termos reais. Esse resultado indica que o Banco Central não considera as informações contidas nas variações do mercado de ações em suas decisões sobre o direcionamento da taxa de juros.

A mesma relação negativa pôde ser constatada ao se analisar as funções de resposta das séries, apesar do impacto do choque de uma variável sobre a outra ser insignificante.

considerada como dependente. 
Figura 2

Função de resposta - IBOVR x TXJR

Resposta à Inovação de Um Desvio Padrão
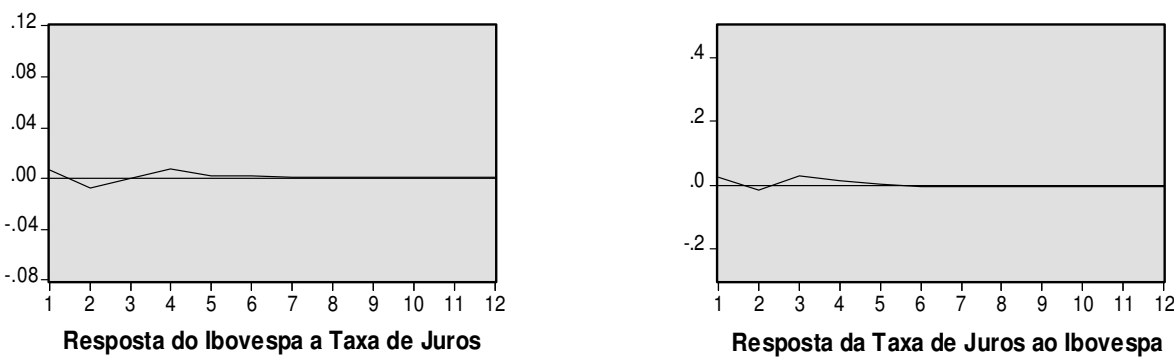

Em relação à interação entre Ibovespa e setor externo, medido pela taxa de câmbio real, constata-se que as inovações do Ibovespa possuem um poder explicativo das variações na taxa de câmbio real. Aproximadamente 19,82\% da variância dos erros de previsão para doze meses da taxa de câmbio real são explicados pelas inovações dos retornos reais do Ibovespa, indicando que as variações dos retornos do Ibovespa podem ser importantes previsores da taxa de câmbio real. Este resultado está de acordo com o encontrado por Nunes et alii (2003), que verificaram causalidade unidirecional do Ibovespa para a taxa de câmbio.

A resposta de um choque inesperado no mercado de ações brasileiro acarreta uma queda de aproximadamente 0,20 pontos percentuais na taxa de câmbio após dois períodos (figura 3). O impacto das variações na taxa de câmbio é mais rapidamente absorvido pelos retornos do Ibovespa, atingindo seu efeito máximo no primeiro e anulando-se próximo ao terceiro período.

Figura 3

Função de resposta - IBOVR x TXCR

\section{Resposta à Inovação de Um Desvio Padrão}
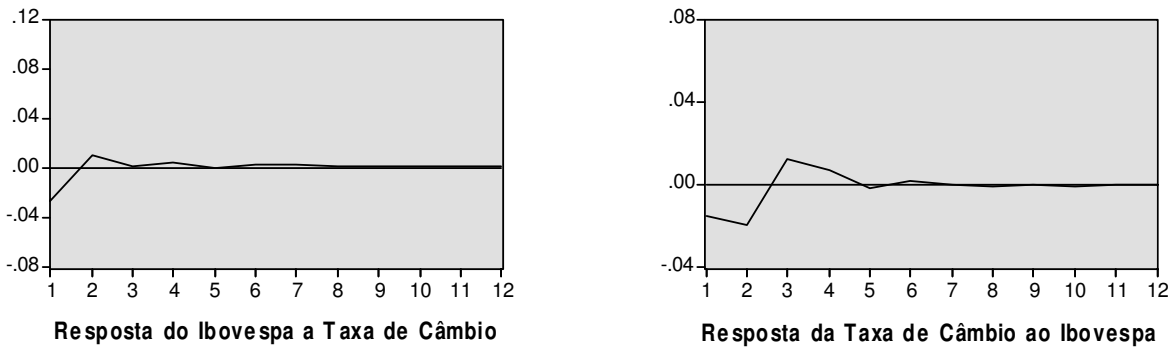
Para a relação entre o mercado de ações e o nível de atividade, pode-se inferir que, ao contrário das suposições prévias, os retornos das ações não servem como um bom previsor das variações no nível de atividade para o caso brasileiro. A influência das inovações no Ibovespa possui um impacto relativamente pequeno sobre a variância dos erros de previsão do PIB (8,83\%). Esses resultados contrariam os de Schwert (1989), Asprem (1989) e Chiang e Doong (1999), cujos estudos indicaram importantes correlações entre as variações contemporâneas do mercado e os níveis futuros da atividade econômica.

As funções de resposta ao impulso do PIB em relação aos choques no Ibovespa indicam uma relação positiva fraca, porém duradoura, entre as variáveis, visto que a mesma persiste mesmo após doze meses (figura 4). Entretanto, apesar do resultado parecer incoerente, ele pode não ser contraditório às suposições prévias, pois considerando que as variações no nível de atividade futuro devam reagir somente aos choques reais se as inovações dos preços no mercado de ações forem decorrentes de choques nominais. As mesmas podem ser completamente absorvidas pelas taxas de desconto, deixando inalterados os fluxos de caixa futuros esperados.

Em contrapartida, para choques do PIB real, a resposta dos retornos de mercado será negativa e persistente (figura 4). Novamente, se os retornos de mercado em termos correntes refletem as expectativas sobre os eventos futuros, é plausível que o mercado de ações não reaja positivamente aos choques não esperados sobre as condições econômicas. Esses resultados estão de acordo com os de Asprem (1989), cujas taxas de crescimento defasadas estão negativamente correlacionadas com os retornos das ações para alguns países europeus, principalmente a Alemanha.

Figura 4

Função de resposta - IBOVR x PIBR

Resposta à Inovação de Um Desvio Padrão
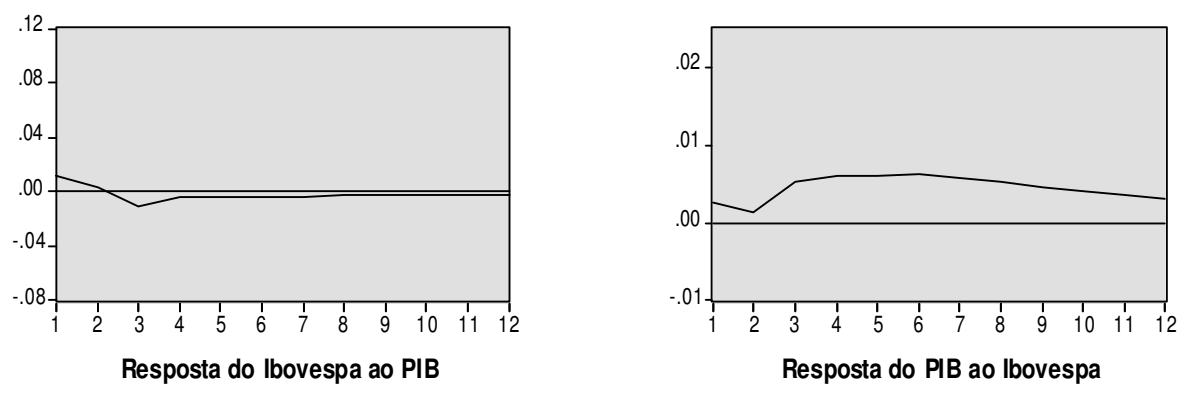
Finalmente, analisando-se a interação dinâmica entre o Ibovespa e o spread do C-bond, observa-se que aproximadamente $54 \%$ da variância dos erros de previsão para doze meses são explicados pelas inovações dos retornos do Ibovespa, indicando que as percepções de risco quanto à economia brasileira seriam representadas, em grande parte, pelas variações do mercado de ações. Este é um resultado interessante, todavia deve ser analisado com cuidado, haja vista que a decomposição de Cholesky (utilizada na decomposição da variância) é sensível à ordenação das variáveis. Portanto, como a variável SCBOND foi ordenada por último, o seu erro de previsão estaria sendo afetado pelos choques nas demais variáveis do sistema. Ordenando-se de maneira inversa, isto é, com os choques do SCBOND exógenos às inovações no Ibovespa, mas com estes sendo afetados pelos primeiros, a relação se inverte com as inovações do SCBOND afetando a variância dos erros de previsão do Ibovespa em aproximadamente 47\%. Esses resultados estão de acordo com os de Nunes et alii (2003), que não constataram qualquer relação causal entre as variáveis utilizando-se séries mensais. De acordo com os autores, isso é decorrente da periodicidade com que as séries foram estudadas e reflete o fato que as séries financeiras apresentam variações de curtíssimo prazo, fazendo com que o impacto seja exercido em um período inferior ao estudado.

Figura 5

Função de resposta - IBOVR x SCBOND

Resposta à Inovação de Um desvio Padrão
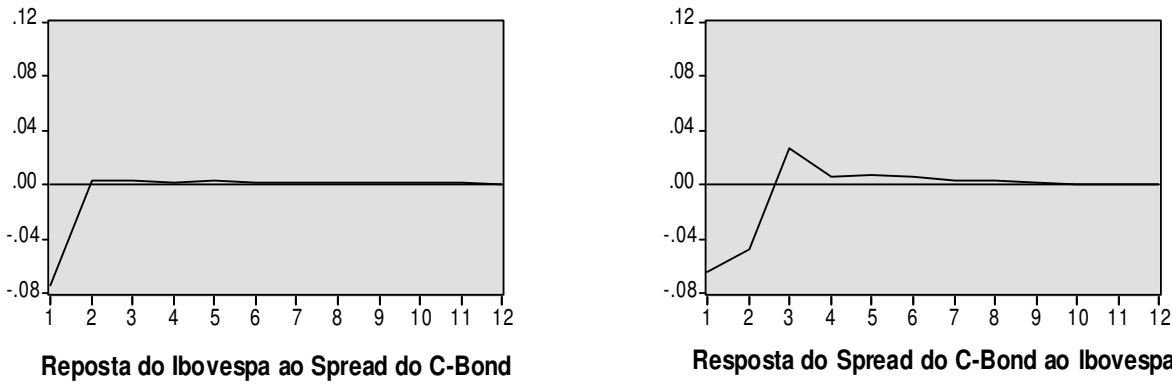

Essa constatação pode ser verificada analisando-se as funções de impulsoresposta das duas séries (figura 5). Utilizando-se um processo de inovação generalizada, a qual não é sensível à ordenação das variáveis, verifica-se que os impactos dos choques sobre ambas as variáveis são significantes e quase que imediatos, indicando que as séries são altamente correlacionadas, porém sem uma efetiva relação causal (no sentido de Granger) entre elas. 


\section{Conclusão}

O presente artigo teve por objetivo realizar um estudo para verificar se há relação entre um conjunto de variáveis macroeconômicas e o retorno dos ativos no mercado de ações brasileiro. Analisou-se, primeiramente, a relação entre a taxa de inflação e os retornos no Ibovespa. Os resultados indicaram para rejeição da "hipótese fisheriana modificada". Ou seja, não se pôde constatar que os retornos do mercado brasileiro sirvam como um hedge contra a taxa de inflação esperada. Rejeitou-se também a hipótese de "proxy effect", na medida em que não se constatou uma relação negativa entre as taxas de inflação e o nível de atividade econômica. Os resultados encontrados tendem a confirmar a suposição inerente à curva de Phillips, da relação positiva entre inflação e a atividade econômica em termos reais. No entanto, a relação negativa entre taxa de inflação e os retornos no mercado brasileiro foi verificada, assim como a relação positiva entre a atividade econômica e os retornos médios do Ibovespa.

O modelo de "causalidade reversa" foi comprovado via modelo de transferência. Os resultados indicaram que as variações no mercado de ações influenciam as variações na taxa de inflação medida pelas variações na taxa selic ex-ante. No entanto, os resultados do componente média rejeitaram a hipótese de Solnik de que a taxa de juros segue um caminho aleatório, isso indica que a taxa de inflação exerce um determinado impacto sobre a taxa de juros real.

Por fim, analisou-se a relação dinâmica entre as variáveis macroeconômicas e o mercado de ações, através da estimativa VAR. Os resultados ratificaram a significativa influência dos retornos de mercado em prever as taxas futuras de inflação. Identicamente, verificou-se uma relação negativa entre a taxa de juros e os retornos de mercado, mesmo que de forma insignificante. Esses resultados indicam que o Banco Central reage às oscilações das variações no mercado por intermédio de alterações nas taxas de juros, mas, muito provavelmente, de forma indireta, como forma de conter possíveis pressões inflacionárias.

Em contrapartida, as variações do índice de mercado e as variações reais no PIB não apresentaram uma relação significativa. Esses resultados contrariam as suposições expostas no modelo de valorização padrão de que o mercado de ações antecipe as variações nos fluxos de caixa futuros esperados e, por conseguinte, os níveis futuros de atividade econômica. 
Em relação ao mercado externo, verificou-se que as variações correntes do Ibovespa têm poder explicativo sobre as variações na taxa de câmbio real. No mesmo sentido, constata-se uma importante relação entre as percepções dos investidores externos sobre a economia brasileira (spreads do C-bond) e os movimentos no mercado de ações.

\section{Referências}

Ajayi, R. A., Friedman, J., \& Mehdian, S. M. (1998). On the relationship between stock returns and exchange rates: Test of Granger causality. Global Finance Journal, 2(9):241-251.

Asprem, M. (1989). Stock prices, asset portfolios and macroeconomic variables in ten European countries. Journal of Banking Finance, 13(4):589-612.

Barro, R. J. (1990). The stock market and investment. Review of Financial Studies, 3(1):115-131.

Bekaert, G., Garcia, M. G. P., \& Harvey, O. R. (1995). The role of capital markets in economic growth. PUC-RJ. Texto Para Discussão n.342.

Blanchard, O. J. (1990). Output, the stock market, and interest rates. American Economic Review, 71(1):132-143.

Campbell, J. Y. (1993). Intertemporal asset pricing without consumption data. American Economic Review, 83(3):487-512.

Chiang, C. T. \& Doong, S. C. (1999). Empirical analysis of real and financial volatilities on the stock excess returns: Evidence from Taiwan industrial data. Global Finance Journal, 10(2):187-200.

Dornbush, R. \& Fischer, S. (1980). Exchange rates and current account. American Economic Review, 70(5):960-971.

Enders, W. (1995). Applied Econometric Time Series. Wiley, New York.

Fama, E. F. (1981). Stock returns, real activity, inflation and money. American Economic Review, 71(4):545-565.

Fama, E. F. (1990). Stock returns, expected returns, and real activity. Journal of Finance, 45(4):1089-1108. 
Fisher, I. (1930). The Theory of Interest. Macmillan, New York.

Gallagher, L. A. \& Taylor, M. P. (2002). The stock return-inflation puzzle revisited. Economics Letters, 75(2):147-156.

Geske, R. \& Roll, R. (1983). The fiscal and monetary linkage between stock returns and inflation. Journal of Finance, 38(1):1-33.

Gutelkin, N. B. (1983). Stock markets returns and inflation: Evidence from the other countries. Journal of Finance, 38(1):49-65.

Hansen, L. P. \& Singleton, K. J. (1983). Stochastic consumption, risk aversion, and the temporal behavior of asset returns. Journal of Political Economy, 91(2):249265 .

Kaul, G. (1987). Stock returns and inflation: The role of monetary sector. Journal of Financial Economics, 18(2):253-276.

Kwon, C. S. \& Shin, T. S. (1999). Cointegration and causality between macroeconomic variables and stock markets returns. Global Finance Journal, 10(1):71-81.

Lee, B. (1992). Causal relations among stock returns, interest rates, real activity, and inflation. Journal of Finance, 42(4):1591-1603.

Lee, W. Y. \& Solt, M. E. (2001). Economic exposure and hysteresis: Evidence from German, Japanese and U. S. stock markets. Global Finance Journal, 12(2):217235.

Mandelker, G. \& Tandon, K. (1985). Common stock returns, real activity, money, and inflation: Some international evidence. Journal of International Money and Finance, 4(2):267-286.

Merikas, A. G. (2002). Stock prices response to real economic variables: The case of Germany. SSRN: Working paper series.

Najand, M. \& Noronha, G. (1998). Causal relations among stock returns, inflation, real activity and interest rates: Evidence from Japan. Global Finance Journal, $9(1): 71-80$.

Nelson, C. R. (1976). Inflation and rates of returns on common stocks. Journal of Finance, 31(2):471-483. 
Nunes, M. S., Costa Jr., N. C. A. D., \& Seabra, F. (2003). Co-integração e causalidade entre as variáveis macroeconômicas, "risco Brasil" e os retornos no mercado de ações brasileiro. Revista de Economia e Administração, 2(3):26-42.

Pesaran, M. H. \& Shin, Y. (1998). Generalised impulse response analysis in linear multivariate models. Economics Letters, 58(1):17-29.

Rigobon, R. \& Sack, B. (2001). Measuring the reaction of monetary policy to the stock market. Cambridge: NBER - Working Papers n.8350, 32 p.

Schwert, G. W. (1989). Why does stock market volatility change over time? Journal of Finance, 44(5):1115-1153.

Shah, H. (1989). Stock returns and anticipated aggregate real activity. University of Chicago.

Sims, C. A. (1980). Macroeconomics and reality. Econometrica, 48(1):1-48.

Solnik, B. (1983). The relations between stock prices and inflationary expectations: The international evidence. Journal of Finance, 38(1):35-48. 